\section{Venöse Thromboembolien beim Mammakarzinom vorhersehbar?}

\begin{abstract}
Venöse Thromboembolien (VTE) sind eine schwerwiegende Komplikation bei malignen Erkrankungen. In einer bevölkerungsbasierten Studie wurden Risiko und klinische Prädiktoren bei Brustkrebspatientinnen untersucht.
\end{abstract}

Dro ro Jahr erleiden 1-2 pro 1.000 Personen eine venöse Thromboembolie (VTE). Bei Brustkrebspatientinnen ist das Risiko etwa 3- bis 4-fach erhöht. Auch wenn das absolute Risiko im Ver-

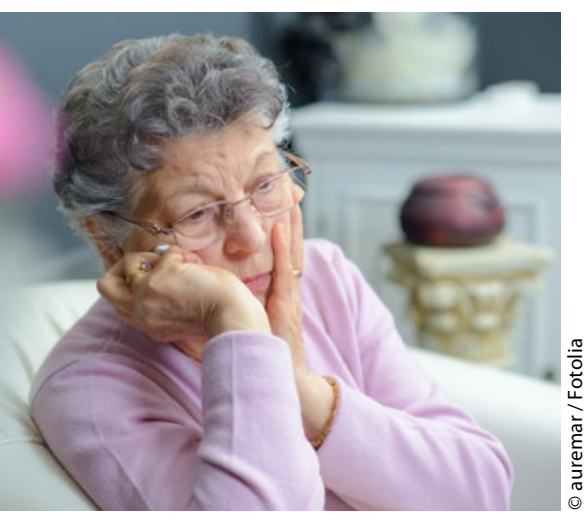

Ein unabhängiger Prädiktor für eine VTE war höheres Lebensalter. gleich zu anderen Krebserkrankungen niedrig ist, können die Konsequenzen doch schwerwiegend und langfristig sein, mit erheblichen Komorbiditäten und Einschränkungen der Lebensqualität v.a. für Patientinnen mit nicht metastasierter Erkrankung und guter Prognose.

In einer bevölkerungsbasierten Studie aus Schweden wurde die Häufigkeit von VTE bei 8.338 Brustkrebspatientinnen (25-75 Jahre), die bei Diagnose keine Fernmetastasen hatten, mit der von 39.013 altersgematchten Kontrollen aus der Allgemeinbevölkerung verglichen.

Nach median 7,2 Jahren hatten 426 Brustkrebspatientinnen ein VTE-Ereignis - eine kumulative Inzidenz von 5, $1 \%$. Diese war gegenüber der Vergleichsgruppe verdreifacht (Hazard Ratio [HR] 3,28, $95 \%$-Konfidenzintervall [95\%-KI] 2,87-3,74). Das relative Risiko für VTE war 6 Monate nach Diagnose am höchs- ten (HR 8,62, $95 \%$-KI 6,56-11,33), anschließend ging es zurück, blieb aber höher als in der Vergleichsgruppe (HR nach 5 Jahren 2,19, $95 \%$-KI 1,80-2,67).

Das mittlere Alter bei Diagnose der VTE war bei den Patientinnen niedriger als bei der Vergleichsgruppe (62,0 vs. 65,3 Jahre). Die kumulative 1-, 2- und 5 -Jahres-Inzidenz von VTE betrug in der Brustkrebskohorte 2,0\%, 2,5\% und $4,0 \%$, in der Vergleichskohorte $0,3 \%$, $0,5 \%$ und $1,1 \%$. Die VTE-Raten lagen in der Brustkrebskohorte bei 7,9, in der Vergleichskohorte bei 2,4 pro 1.000 Personenjahren. Unabhängige Prädiktoren einer VTE waren höheres Lebensalter, Übergewicht, VTE in der Vorgeschichte, Komorbiditäten, Tumorgröße $>40 \mathrm{~mm}$, Progesteronrezeptor(PR)-negativer Status, $>4$ befallene Lymphknoten und erhaltene Chemo- und endokrine Therapie.

Fazit: Die Daten bestätigen, dass Brustkrebspatientinnen langfristig ein erhöhtes VTE-Risiko haben. Prädiktoren für ein solches Ereignis konnten identifiziert werden.

Kathrin von Kieseritzky

Brand JS et al. Time-Dependent Risk and Predictors of Venous Thromboembolism in Breast Cancer Patients: A Population-Based Cohort Study. Cancer. 2017;123(3):468-75.

\section{Topotecan beim platinsensitiven Ovarialkarzinom}

\author{
In einer randomisierten Phase-III-Studie wurden die Effizienz und Sicherheit \\ von Topotecan und Carboplatin bei platinsensitiven rezidivierten Ovarialkar- \\ zinomen mit der von platinbasierten Standardkombinationen verglichen.
}

$B^{\mathrm{e}}$ ei Patientinnen mit rezidiviertem platinsensitivem Ovarialkarzinom werden verschiedene platinbasierte Regimes empfohlen. Der Topoisomerase-I-Inhibitor Topotecan ist in der palliativen Therapie gut etabliert und bei platinresistenten Tumoren ähnlich effizient wie Paclitaxel und pegyliertes liposomales Doxorubicin. In der Studie erhielten insgesamt 550 Patientinnen randomisiert Topotecan/Carboplatin (TC) oder ein Standardregime mit Paclitaxel/Carboplatin (PC), Gemcitabin/Carboplatin (GC) oder pegyliertem liposomalem Doxorubicin/Carboplatin (PLDC). Primärer Endpunkt war das progressionsfreie
Überleben (PFS) nach 12 Monaten, sekundäre Endpunkte u.a. das Gesamtüberleben (OS) und die Ansprechrate.

Nach median 20 Monaten betrug die Rate für das 12-Monats-PFS 37,0\% im TC- und 40,2\% im Standardarm $(p=0,470)$. Auch abhängig vom Alter, der Zahl der Vortherapien und der Histologie (seröse bzw. nichtseröse Tumoren) ergaben sich keine Unterschiede. Insgesamt starben 262 (47,6\%) Patientinnen, die Krankheit war bei 426 (77,5\%) progredient. Die generelle Ansprechrate betrug 73,1\% im TC-Arm und 75,1 \% im Standardarm ( $p=0,149)$. Das mediane PFS lag im TC-Arm bei 10, im Standardarm bei 11 Monaten $(\mathrm{p}=0,414)$. Insgesamt betrug das mediane OS 27 Monate, im TC-Arm 25, im Standardarm 31 Monate, auch hier gab es keinen signifikanten Unterschied ( $\mathrm{p}=0,163)$. Hämatologische Toxizitäten vom Grad 3/4 waren im Standardarm signifikant häufiger als im experimentellen $\operatorname{Arm}(\mathrm{p}<0,001)$.

Fazit: Bei platinsensitiven rezidivierten Ovarialkarzinomen war die Kombination aus Topotecan plus Carboplatin gegenüber Standardregimes zwar besser verträglich, konnte aber weder PFS noch OS verbessern.

Kathrin von Kieseritzky

Sehouli J et al. Topotecan plus carboplatin versus standard therapy with paclitaxel plus carboplatin (PC) or gemcitabine plus carboplatin (GC) or pegylated liposomal doxorubicin plus carboplatin (PLDC): a randomized phase III trial of the NOGGO-AGO-Study Group-AGO Austria and GEICOENGOT-GCIG intergroup study (HECTOR). Ann Oncol. 2016;27(12):2236-41. 